\title{
MICROSCOPIC DERIVATION OF MACROSCOPIC VAN DER WAALS FORCES
}

\author{
M.J.RENNE and B.R. A. NIJBOER \\ Instituul voor theoretische fi'sica. Rijksuniversiteit Utrecht. The Netherlands
}

Received 15 September 1967

\begin{abstract}
For a general system of isotropic harmonic oscillators with non-retarded dipole interaction a formula for the interatomic forces is derived. It is used to give an atomistic derivation of macroscopic van der Waals forces in terms of the dielectric constant.
\end{abstract}

\section{INTRODUCTION}

It is well-known that neutral macroscopic bodies, when placed at a distance of the order of $1000 \ddot{A}$, attract each other as a result of the Van der Waals forces between their atoms. However, the resulining force is not just the sum of the Van der Waals forces between pairs of atoms, but there are deviations due to many-body effects. A macroscopic theory for this phenomenon has been developed by Lifshicz [ 1$]$ by introducing fluctuating terms in the Maxwell-equations for a dielectric inedium. For the force per unit area between two semi-infinite media a distance $d$ apart Lifshitz finds in the case that $d \ll \lambda(\lambda$ is the principal absorption wave length of the material)

$$
K(d)=-\frac{\pi}{16 \pi^{2} d^{3}} \int_{0}^{\infty} \mathrm{d} \omega \int_{0}^{\infty} \mathrm{d} x x^{2}\left\{\left(\frac{\epsilon(\mathrm{i} \omega)+1}{\epsilon(\mathrm{i} \omega)-1}\right)^{2} \mathrm{e}^{x}-1\right\}^{-1}
$$

where $\epsilon(\omega)$ is the frequency-dependent dielectric constant of the medium.

In this note we will give an atomistic derivation of the Van der Waals interaction between macroscopic bodies, starting from the Lorentz-model of an atom (also known as the Drude-model), i.e. we suppose that the atom is represented by an electron oscillating harmonically around a positively charged equilibrium position.

\section{INTERATOMIC FORCES FOR AN ARBITRARY SYSTEM OF HAFIVIONIC CSCILLATORS WITH DI-} POLE INTERACTION

Consider a system of $N$ isotropic oscillators, the equilibrium positions of which are fixed at $r_{1}$, $r_{2}, \ldots, r_{N}$. The deviation of the $i$ th oscillator from its equilibrium is called $u_{i}$. The "atoms" are supposed to interact with the non-retarded dipole-dipole interaction. The Hamiltonian of the system is given by

$$
H=\sum_{i=1}^{N} \frac{p_{i}^{2}}{2 m}+\sum_{i=1}^{N} \frac{2}{2} m \omega_{0}^{2} u_{i}^{2}+\frac{1}{2} e^{2} \sum_{i, j=1}^{N} \sum_{\alpha, \beta=1}^{3} T_{i j}^{\alpha \beta} u_{i}^{\alpha} u_{j}^{\beta},
$$

where

$$
T_{i j}^{\alpha \beta}=\left\{\nabla_{i} \nabla_{j}\left|r_{i}-r_{j}\right|^{-1}\right\}^{\alpha \beta}=\left|r_{i}-r_{j}\right|^{-3}\left\{\delta_{\alpha \beta}-3\left(r_{i}^{\alpha}-r_{j}^{\alpha}\right)\left(r_{i}^{\beta}-r_{j}^{\beta}\right)\left|r_{i}-r_{j}\right|^{-2}\right\} \quad \text { if } i \neq j ; T_{i i}^{\alpha \beta}=0 ;
$$

$\alpha$ and $\beta$ number the cartesian coordinates. The quadratic hamiltonian can be diagonalized by an orthogonal transformation. Let the eigenfrequencies be $\omega_{k}(k=1,2, \ldots, 3 N)$ and let $\lambda_{k}$ be the eigenvalues of the $3 N \times 3 N$ matrix $\mathcal{J}$, which is built up from the $T_{i j}$. Then 
with

$$
\omega_{k}=\omega_{0}\left(1-\sum_{n=1}^{\infty} a_{n}\left(-\alpha_{0}\right)^{n_{2}^{n}}\right)
$$

$$
a_{n}=\frac{(2 n) !}{(2 n-1)(n !)^{2} 2^{2 n}} .
$$

The interaction energy between the atoms when the system in its electronic ground state (Born-Oppenheimer approximation) is given by

$$
E\left(r_{1}, r_{2}, \ldots, r_{N}\right)=\frac{1}{2} \hbar \sum_{k=1}^{3 N}\left(\omega_{k}-\omega_{0}\right)=-\frac{1}{2} \hbar \omega_{0} \sum_{n=2}^{\infty} a_{n}\left(-\alpha_{0}\right)^{n} \operatorname{Tr}\left(\mathcal{T}^{n}\right),
$$

where use has been made of the weil-known theorem that

$$
\sum_{k=1}^{3 N} \lambda_{k}^{n}=\operatorname{Tr}\left(\mathcal{T}^{n}\right)
$$

Now $\operatorname{Tr}\left(\mathcal{T}^{n}\right)$ can again be expressed in terms of the $3 \times 3$ matrices $T_{i j}$ according to

$$
\operatorname{Tr}\left(\mathcal{T}^{n}\right)=\sum_{i_{1}, \ldots, i_{n}=1}^{N} \operatorname{Tr}\left\{T_{i_{1} i_{2}} \cdot T_{i_{2} i_{3}} \cdots T_{i_{n^{i}} i}\right\}
$$

in particular

$$
\begin{aligned}
& \operatorname{Tr}(\mathscr{T})=0 \\
& \operatorname{Tr}\left(\mathscr{T}^{2}\right)=\sum_{i, j=1}^{N} \operatorname{Tr}\left\{T_{i j} \cdot T_{j i}\right\}=\sum_{i \neq j=1}^{N} \frac{6}{\left|r_{i}-r_{j}\right|^{6}} .
\end{aligned}
$$

We find therefore

$$
\begin{aligned}
& E\left(r_{1}, r_{2}, \ldots, r_{N}\right)=-\frac{3}{8} \pi \omega_{o} \alpha_{o}^{2} \sum_{i \neq j=1}^{N} \frac{1}{\left|r_{i}-r_{j}\right|^{6}}
\end{aligned}
$$

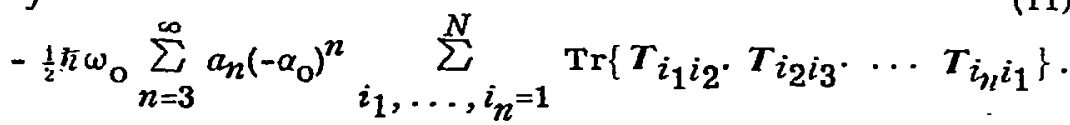

This result gives a very general answer to a problem discussed by Sparnaay [2], i.e. the deviations from additivity of Van der Waals forces. The first term of (11) represents the familiar non-retarded Van der Waals interaction between the atoms taken pairwise, the term with $n=3$ describes the 3 -particle interactions, etc. The successive terms of (11) can be visualized by means of diagrams in such a way that each factor $T$ is represented by a line. For a macroscopic system (gas, liquid, crystai) these contributions may in principle be expressed in terms of many-particle distribution functions and their relative importance cstimated *.

\section{INTERACTION BETWEEN AN ATOM AND A SEMI-INFINITE MEDIUM.}

We will now apply (11) to the case of the interaction between one atom at a distance $d$ from a semiinfinite homogeneous mcditum consisting of the same kind of atoms. We number the atoms of the medium

* After this note had been written a paper by Lucas [3] appeared, where for the case of crystals formula (7) is dexived and where numerical calculations of its successive terms are given. 


$$
\begin{array}{rlr} 
& =0 & \text { otherwise; } \\
\text { and } v_{i j}^{\alpha \beta} & =i \delta_{i, 0}-\delta_{j, 0} \mid T_{i j}^{\alpha \beta},
\end{array}
$$

we find, applying (7) twice, for the interaction between atom $D$ and the medium

$$
U(d)=\frac{1}{2} \hbar \sum_{k=1}^{3(N+1)}\left(\Omega_{k}-\omega_{k}\right)=-\frac{1}{2} \hbar \omega_{0} \sum_{n=2}^{\infty} a_{n}\left(-\alpha_{0}\right)^{n} \operatorname{Tr}\left\{(\hat{\mathcal{T}}+v)^{n}-\hat{\mathfrak{T}}^{n_{1}}\right\} .
$$

One may show very simply that, when terms in $v$ of order 4 and higher are neglected (because they decrease with a higher power of the distance $d$ than the terms in $\left.v^{2}\right),(13)$ reduces to

$U(d)=-\frac{1}{2} \pi \omega_{0} \sum_{n=2}^{\infty} n a_{n}\left(-\alpha_{0}\right)^{n} \sum_{i_{1}}, \ldots, i_{n-1}^{N} \operatorname{Tr}\left\{T_{i_{1} i_{2}} \cdot T_{i_{2} i_{3}} \ldots T_{i_{n-2}, i_{n-1}} \cdot T_{i_{n-1} \mathrm{o}} \cdot \boldsymbol{T}_{\mathrm{o} i_{1}}\right\}$.

The first term of (14), i.e. the term with $n=2$, is

$$
-\frac{1}{8} \pi \omega_{0} \alpha_{0}^{2} \sum_{i=1}^{N} \operatorname{Tr}\left\{T_{o i}^{2}\right\}=-\frac{\pi \hbar \omega_{0} \rho \alpha_{0}^{2}}{8 d^{3}},
$$

where the summation is replaced by an integration over the medium and where $\rho$ is the number density. It gives the additive part of the interaction, i.e. the sum of the Van der Waals interactions between the one atom and the atoms of the medium. The terms with $n=3,4$ etc. give the correction to this elementary result as a consequence of 3-particle, 4-particle, etc. interactions. They will be evaluated in the following.

\section{EVALUATION OF (14) IN TERMS OF THE DIELECTRIC CONSTANT}

Let us consider the case of a dipole oscillator $\mu_{\mathrm{o}}(t)=\mu_{\mathrm{o}} \mathrm{e}^{\mathrm{i} \omega t}$ at a distance $d$ from our semi-infinile medium. We then have the equations $(i=1,2, \ldots, N)$

$$
\begin{aligned}
\mu_{i}(t) & =-\alpha(\omega) T_{i 0} \cdot \mu_{0}(t)-\alpha(\omega) \sum_{j=1}^{N} T_{i j} \cdot \mu_{j}(t)= \\
& =-\alpha(\omega) T_{i 0} \cdot \mu_{0}(t)+\alpha^{2}(\omega) \sum_{j=1}^{N} T_{i j} \cdot T_{j 0} \cdot \mu_{0}(l)-\ldots
\end{aligned}
$$

by iteration. Here $\alpha(\omega)=e^{2 / m}\left(\omega_{0}^{2}-\omega^{2}\right)$ is the polarigability at frequency $\omega$ of the oscillators. Now from macroscopic electrostatics it is known that in such a situation the macroscopic electric field inside the medium is given by [4]

$$
E_{\omega}\left(r_{i}\right)=-\frac{3}{\epsilon(\omega)+1} T_{i 0} \mu_{0}(t)
$$

Substituting now into (16)

$$
\mu_{i}(t)=\frac{1}{\rho} P_{\omega}\left(r_{i}\right)=\frac{1}{2 \pi \rho} \frac{\epsilon(\omega)-1}{\epsilon(\omega)+1} T_{i 0 .} \mu_{0}(t),
$$


one can show after some algebra that the interaction (14) between atom and medium can be written as

$$
U(d)=-\frac{\hbar}{4 \pi d^{3}} \int_{0}^{\infty} \alpha(i \omega) \frac{\epsilon(i \omega)-1}{\epsilon(i \omega)+1} \mathrm{~d} \omega .
$$

This resuli also follows exactly from Lifshitz' macroscopic formula (1), if it is applied to the case that one of both media is rarefied. For the case of one atom outside a dielectric medium (and for our Farticular atom model) we therefore have succeeded in giving an atomistic calculation of the Van der Waals interaction, taking into account many-particle interactions.

\section{A SIMPLE APFROXIMATE FORMULA}

The formula (19) takes account of the complete series expansion (14). We will now derive an approximate formula by evaluating only the term $n=3$ of (14), that is the first correction to additivity (eq. (15)) due to 3-particle interactions. From (16), (17) and (18) it is easily found that

$$
\alpha_{o} \sum_{i, j=1}^{N} \operatorname{Tr}\left\{T_{i j} \cdot T_{j o} \cdot T_{o i}\right\}=\left\{2 \pi \rho \alpha_{o} \frac{\epsilon_{o^{+1}}}{\epsilon_{o^{-1}}}-1\right\} \sum_{i} \operatorname{Tr}\left\{T_{o i}^{2}\right\} .
$$

where $\epsilon_{0}$ is the static dielectric constant. Hence, introducing (20) into (14), we find the approximate result (2-lines and 3-lines diagrams only)

$$
U(d)=-\frac{\pi \hbar \omega_{0} \rho \alpha_{0}^{2}}{8 d^{3}}\left\{1-\left(\frac{3}{2} \pi \rho \alpha_{0} \frac{\epsilon_{\mathrm{o}}+1}{\epsilon_{\mathrm{o}}-1}-\frac{3}{4}\right)\right\} .
$$

The term which is to be subtracted from 1 gives the (approxinate) correction to additivity. To estimate its order of magnitude let "a assume that the law of Clausius-Mossotti holds. Then the correction term is

$$
-\frac{3}{8} \frac{\epsilon_{\mathrm{o}}-1}{\epsilon_{\mathrm{o}}+2} \text {. }
$$

If in the case of two semi-infinite media a distance $d$ apart we again include in addition to the elementary pair interaction the correction due to 3-line diagrams only, we find twice the correction calculated above. That is, the force per unit area is in this case approximately

$$
K(d) \simeq-\frac{\pi \hbar \omega_{0} \rho^{2} \alpha_{o}^{2}}{8 d^{3}}\left\{1-\left(3 \pi \rho \alpha_{0} \frac{\epsilon_{0}+1}{\epsilon_{0}-1}-\frac{3}{2}\right)\right\}
$$

Assurning again the Clausius-Mossotti law the correction term is now

$$
-\frac{3}{4} \frac{\epsilon_{o^{-1}}}{\epsilon_{\mathrm{o}^{+2}}}
$$

i.e. for $\epsilon_{0}=1.5$ it is $-11 \%$, for $\epsilon_{0}=2$ it is $-19 \%$, for $\epsilon_{0}=3$ it is $-30 \%$. A more detailed report will be published elsewhere.

\section{ACKNOWLEDGEMENT}

We wouid like to thank Mr. 15. Schram for useful discussions.

\section{REFERENCES}

[1] E. M. Lifshitz, Soviet Phys. -JETP 2 (1956) 73:

cf. also L. Landau and E. M. Lifshitz, Electrodynamics of Continuous Media (Pergamon Press, Oxford, 1960) p. 90.

[2] M. J. Sparnaay, Physica 25 (1959) 217.

[3] A. Lucas, Physica 35 (1967) 353.

[4] Cf. e.g. J.D. Jackson, Classical Electrodynamics (Wiley, New York, 3rd ed.) p. 110. 